\title{
ESTUDO DO DESENVOLVIMENTO DA FUNÇÃO MOTORA AOS 8 E 12 MESES DE IDADE EM CRIANÇAS NASCIDAS PRÉ-TERMO E A TERMO
}

\author{
Marisa C. Mancini'1, Silvana Teixeira², Louise G. de Araújo², Maria Lúcia Paixão3, \\ Lívia de Castro Magalhães ${ }^{4}$, Zélia Araújo Cotta Coelho ${ }^{5}$, Ana Paula Benseman Gontijo ${ }^{6}$, \\ Sheyla Rossana Cavalcanti Furtado ${ }^{7}$, Rosana Ferreira Sampaio ${ }^{8}$, \\ Sérgio Teixeira da Fonseca9
}

\begin{abstract}
RESUMO - Objetivo: Comparar o desenvolvimento da função motora de crianças nascidas pré-termo com crianças nascidas a termo, aos 8 e 12 meses de idade. Investigar a relação entre a qualidade motora aos 8 meses e a habilidade motora aos 12 meses. Método: Trinta e duas crianças participaram deste estudo: 16 nascidas prétermo (grupo de risco) e 16 nascidas a termo (grupo controle). A movimentação espontânea das crianças foi avaliada aos 8 meses e as habilidades e independência em mobilidade foram avaliadas aos 12 meses de idade (idades corrigidas para grupo pré-termo), utilizando-se testes infantis padronizados (AIMS e PEDI, respectivamente). Os dados foram analisados através dos textest de Student para grupos independentes (comparação entre grupos) e de correlação de Pearson (comparação intra grupo). Resultados: Não foi evidenciada diferença significativa na comparação de crianças nascidas a termo com as pré-termo nem aos 8 nem aos 12 meses de idade. No grupo controle, foi observada relação significativa $(r=0,67 ; p=0,004)$ entre movimentação aos 8 meses e habilidade de mobilidade aos 12 meses. No grupo de risco, houve relação significativa entre habilidade e independência em mobilidade aos 12 meses de idade corrigida $(r=0,80$; $\mathrm{p}=0,0001$ ). Conclusão: Na ausência de outros distúrbios e com correção da idade em pré-termos, 0 desenvolvimento motor pode ser semelhante ao de crianças nascidas a termo. A forma pela qual as crianças nascidas pré-termo adquirem suas habilidades funcionais parece ocorrer de modo diferente da observada em crianças a termo.
\end{abstract}

PALAVRAS-CHAVE: prematuro, desenvolvimento infantil, atividade motora.

\begin{abstract}
Study of motor function at $\mathbf{8}$ and $\mathbf{1 2}$ months of age in preterm and at term children
ABSTRACT - Objective: To compare the development of motor function in children born preterm with those born at term, at 8 and 12 months of age. To investigate the relation of motor function quality at the age of 8 months with motor ability at 12 months. Method: Thirty-two children participated in this study: 16 were born preterm (risk group) and 16 were born at term (control group). The spontaneous movements of the children were assessed at 8 months and their mobility skills and independence were assessed at 12 months (corrected ages for the preterm group), using standardized developmental tests (AIMS and PEDI, respectively). Data were analysed using independent t-tests (between-group comparison) and Pearson correlation coefficients (within-group comparison). Results: There was no significant difference in motor function, between those born preterm with those born at term, either at 8 or at 12 months of age. In the control group, there was significant association $(r=0.67 ; p=0.004)$ between movement at 8 months and mobility skills at 12 months. In the risk group, there was significant relationship between skills and independence in mobility, at 12 months corrected age $(r=0.80 ; p=0.0001)$. Conclusion: Preterm born children, without other disorders and with age correction, might show a similar motor development as those born at term. The path for the acquisition of motor abilities in preterm born children appears to differ among those infants.
\end{abstract}

KEY WORDS: premature infant, child development, motor activity.

\footnotetext{
Escola de Educação Física, Fisioterapia e Terapia Ocupacional (EEFFTO), Universidade Federal de Minas Gerais (UFMG): ${ }^{1}$ Doutora em Ciências, Professora Adjunta, Departamento Terapia Ocupacional (DTO); ${ }^{2}$ Fisioterapeuta, Bolsista de Iniciação Científica do CNPq; ${ }^{3}$ Especialista, Professora Assistente, Departamento Fisioterapia (DFT); ${ }^{4}$ Doutora em Educação, Professora Adjunta, DTO; ${ }^{5}$ Especialista, Professora Auxiliar, DTO; ${ }^{6}$ Especialista, Professora Auxiliar, DFT; ${ }^{7}$ Mestre em Ciências, Professora Assistente, DFT; ${ }^{8}$ Doutora em Saúde Pública, Professora Adjunta, DFT; ${ }^{9}$ Doutor em Ciências, Professor Adjunto, DFT. Fontes financiadoras deste estudo: Fundação de Amparo à Pesquisa do Estado de Minas Gerais (FAPEMIG); Pró-Reitoria de Pesquisa da Universidade Federal de Minas Gerais e Conselho Nacional de Desenvolvimento Científico e Tecnológico (CNPq).
}

Recebido 20 Fevereiro 2002, recebido na forma final 17 Maio 2002. Aceito 7 Junho 2002.

Dra. Marisa Cotta Mancini - Rua Minerva 556 /602 - 30720-580 Belo Horizonte MG - Brasil. FAX: 3134994790.

E-mail: mcmancini@metalink.com.br 
O aumento na expectativa de sobrevivência de recém-nascidos pré-termo, com idade gestacional inferior a 37 semanas e com peso ao nascimento menor ou igual a $2500 \mathrm{~g}$, tem sido atribuído a melhora dos cuidados neonatais ${ }^{1-3}$. A literatura relata que 0 índice mundial de recém-nascidos com baixo peso varia de 6 a 11\%2. Nos Estados Unidos, a taxa de nascimento pré-termo equivale a cerca de $10 \%$ dos nativivos; o índice de mortalidade destas crianças sofreu decréscimo em torno de $50 \%$ nos últimos anos $^{4,5}$. No Brasil, de acordo com dados apresentados na 5a Jornada Materno-Infantil (1998) a taxa de nascimento pré-termo representa $11 \%$ dos nascimentos $^{6}$. Neste grupo de neonatos, observa-se aumento na incidência de distúrbios neuromotores entre os sobreviventes 7,8 , o que tem estimulado o surgimento de programas de acompanhamento destas crianças, que são consideradas de risco para distúrbios ou atraso no desenvolvimento ${ }^{2,9-11}$. O desenvolvimento neuropsicomotor da criança é aspecto importante do desenvolvimento infantil. As aquisições motoras no primeiro ano de vida são fator relevante no prognóstico do desenvolvimento global da criança, pois o período compreendido entre o nascimento e o final do primeiro ano de vida é considerado como um dos mais críticos no desenvolvimento infantil $^{3}$. Neste período, o desenvolvimento motor apresenta ritmo acelerado de mudanças que culminam nas funções de mobilidade, com a aquisição do engatinhar e da marcha independente, respectivamente aos 9 e 12 meses de idade 3 . Fatores de risco, como nascimento pré-termo e baixo peso, podem interferir no ritmo e nos padrões motores destas aquisições durante o primeiro ano de vida da criança ${ }^{11,12}$.

Grande parte da literatura sobre o desenvolvimento de crianças pré-termo tem centrado suas investigações na relação entre fatores de risco ao nascimento e componentes neuromotores do desenvolvimento ${ }^{1,2,11-20}$. Os resultados destes estudos têm revelado que crianças nascidas pré-termo e com baixo peso diferem daquelas nascidas a termo e com peso adequado, em relação ao tônus muscular, reflexos primitivos e reações posturais, principalmente nos primeiros meses de vida ${ }^{1,15,18,19}$. Entretanto, a influência destes componentes neuromotores na aquisição de funções motoras mais complexas ainda não está bem documentada. Allen e Capute ${ }^{14}$ investigaram a relação entre exame neurológico realizado no período neonatal e o surgimento de anormalidades do desenvolvimento no primeiro ano de vida. Estes autores argumentam que exames neurológicos baseados em componentes e marcos básicos do desenvolvimento neuromotor têm pequeno valor predi- tivo no prognóstico funcional do desenvolvimento infantil14,18. Este tipo de argumentação ilustra uma discussão mais ampla referente à mudança de paradigma nesta área de conhecimento. Tradicionalmente, avaliações de crianças consideradas de risco para apresentar distúrbios neuromotores priorizava a identificação de fatores anormais do desenvolvimento, como variações de tônus muscular e persistência de reflexos primitivos. Atualmente, a ênfase está na documentação do desempenho funcional da criança, salientando sua movimentação espontânea no ambiente ${ }^{21-24}$. Este novo paradigma tem influenciado e motivado a produção científica da área, promovendo o desenvolvimento de novos testes e avaliações.

Estudos atuais têm revelado que as diferenças no desempenho motor de crianças pré-termo e a termo são observadas não só no que se refere aos componentes neuromotores, mas também na qualidade da função motora. A observação sistemática da qualidade dos movimentos espontâneos de crianças de risco é fator importante na identificação precoce de disfunções do desenvolvimento ${ }^{11,14,21}$. Prechtl et al. ${ }^{23}$ compararam os resultados de uma avaliação observacional de movimentos espontâneos com os de uma avaliação neurológica tradicional em crianças de risco e concluíram que observações da movimentação espontânea apresentaram maior valor preditivo no prognóstico do desenvolvimento destas crianças. Mais recentemente, novas abordagens de avaliação têm sido desenvolvidas com base nestas tendências teóricas ${ }^{5,22,25,26}$. Estas avaliações apresentam-se como testes padronizados que documentam de forma objetiva e quantitativa tanto a movimentação espontânea quanto outras características funcionais da criança, possibilitando assim a investigação sistemática de aspectos como a qualidade do movimento e a mobilidade, que até então eram abordados de forma indireta e subjetiva.

Seguindo a corrente atual mais voltada para análise de padrões funcionais, o objetivo deste estudo foi comparar o desenvolvimento da função motora em crianças nascidas pré-termo e a termo, aos 8 e 12 meses de idade. Além disso, este estudo investigou a relação entre movimentação aos 8 meses e mobilidade aos 12 meses de idade em cada um dos grupos.

\section{MÉTODO}

Participantes

Dois grupos de 16 crianças participaram deste estudo. O grupo de risco incluiu crianças nascidas pré-termo com idade gestacional menor que 34 semanas ecom peso menor ou igual a 2500 gramas ao nascimento. Os critéri- 
os de exclusão para a participação destas crianças no estudo foram: presença de distúrbios associados como retardo mental, problemas neurológicos ou ortopédicos e presença de distúrbios sensoriais (visuais ou auditivos) e/ ou sinais de padrões anormais do desenvolvimento neuromotor. O grupo controle foi composto por crianças nascidas a termo que apresentaram desenvolvimento normal, sem atraso ou distúrbio evidenciado, e que não faziam uso sistemático de medicação.

A seleção das crianças de ambos os grupos foi não aleatória com base nos critérios descritos acima. O grupo de risco foi constituído por crianças que frequentam o Ambulatório de Acompanhamento da Criança de Risco (ACRIAR) que funciona no complexo do Hospital das Clínicas da Universidade Federal de Minas Gerais (UFMG). $O$ ACRIAR é um serviço assistencial composto por equipe interprofissional que acompanha e avalia sistematicamente o crescimento eo desenvolvimento de crianças pré-termo nascidas na maternidade do Hospital das Clínicas da UFMG. Este serviço acompanha crianças a partir do primeiro mês de idade corrigida até sete anos de idade. As crianças do grupo controle foram selecionadas em creches da região metropolitana de Belo Horizonte. As crianças dos dois grupos apresentaram equivalência de sexo enível sócio-econômico da família. Características sócio-econômicas foram determinadas conforme critério proposto pela Associação Brasileira de Institutos de Pesquisa de Mercado (ABIPEME) ${ }^{27}$. Crianças de ambos os grupos foram avaliadas aos 8 e 12 meses de idade (corrigida para o grupo prétermo).

\section{Instrumentação}

Este estudo utilizou duas avaliações padronizadas que documentam aquisições funcionais do desenvolvimento infantil: Alberta Infant Motor Scale (AIMS)22 e Pediatric Evaluation of Disability Inventory (PEDI) ${ }^{25}$. Ambastêm sido muito utilizadas tanto na sistematização da prática clínica quanto no desenvolvimento de pesquisas nos Estados Unidos, Canadá e Europa.

O AIMS ${ }^{22}$ éum teste predominantemente observacional que foi desenvolvido para avaliar aquisições motoras de crianças do nascimento até os 18 meses de idade. Este teste se propõe a documentar longitudinalmente o desenvolvimento motor de crianças que apresentam risco para atraso nas aquisições motoras. O teste consiste de 58 itens agrupados em quatro subscalas que descrevem o desenvolvimento de movimentação espontânea e de habilidades motoras em posições básicas, incluindo prono, supino, sentado e de pé. Durante a avaliação, o examinador deve observar a movimentação da criança em cada uma das posições básicas, levando em consideração aspectos do desempenho motor tais como descarga de peso, postura e movimentos antigravitacionais. A aplicação do teste leva em média 20 minutos. $O$ escore consiste em uma escolha dicotomizada para cada item que deve ser avaliado como observado ou não observado. Cada item observado no repertório das habilidades motoras da criança recebe escore 1 (um) e cada item não observado recebe escore 0 (zero). Os itens observados em cada uma das subscalas são somados resultando em quatro subtotais (prono, supino, sentado e de pé). O escore total do teste é dado pela soma dos subtotais obtidos em cada subscala. Este escore total pode ser convertido em percentil de desempenho motor estabelecido com base na amostra normativa do teste ${ }^{5,22,28}$. Jeng et al. ${ }^{5}$ ao utilizar este teste em crianças pré-termo concluíram que as medidas obtidas com o AIMS possuem altos valores de confiabilidade e validade neste grupo de risco ${ }^{5,29}$. O teste AIMS foi utilizado para avaliar a movimentação espontânea das crianças aos 8 meses de idade (corrigida para crianças do grupo de risco).

O teste PEDI ${ }^{25}$ é uma avaliação baseada em julgamento, realizada através de entrevista estruturada com os pais ou responsáveis pela criança. Este teste foi recentemente traduzido para o português ${ }^{30} \mathrm{e}$ adaptado para contemplar as especificidades sócio-culturais do Brasil, com permissão e colaboração dos autores da avaliação original. $O$ teste PEDI descreve o perfil funcional de crianças na faixa etária entre 6 meses e 7 anos e meio de idade. O perfil documentado pelo PEDI informa sobre três aspectos importantes do desenvolvimento funcional que são as habilidades presentes no repertório da criança, a independência no desempenho de atividades diárias e as modificações do ambiente utilizadas para facilitar o desempenho funcional. Cada um destes três aspectos caracteriza uma parte da avaliação.

A primeira parte informa sobre as habilidades que a criança apresenta para desempenhar atividades e tarefas do cotidiano em três áreas de função: auto-cuidado (inclui 73 itens ou atividades funcionais), mobilidade (inclui 59 itens ou atividades funcionais) e função social (inclui 65 itens ou atividades funcionais). Os itens desta parte são avaliados com escore 1, se a criança for capaz de desempenhar a atividade em sua rotina diária, ou 0 , se a criança não for capaz de desempenhar a atividade funcional. Os escores obtidos pela criança são somados em cada área de função, resultando em três escores totais de habilidades (auto-cuidado, mobilidade e função social).

A segunda parte do teste estima a quantidade de ajuda que a criança recebe do cuidador para realizar 20 tarefas funcionais divididas nas mesmas três áreas de função. Nesta parte da avaliação, a quantidade de assistência é avaliada em escala ordinal que varia de 0 (necessita de assistência total) a 5 (a criança é independente no desempenho funcional) com graduações intermediárias indicando níveis diferenciados de assistência (máxima, moderada, mínima ou supervisão). Da mesma forma que na parte l, os escores recebidos em cada item são somados, obtendo-se um escore total de independência da criança para cada área de função (auto-cuidado, mobilidade e função social). $O$ teste PEDI documenta ainda a frequência de modificações do ambiente utilizadas no desempenho de tarefas funcionais nas três áreas descritas acima. O escore total bruto obtido em cada escala pode ser transformado em escore normativo (percentil de desempenho) e em 
escore contínuo obtido através de metodologia específica de transformação (Rasch) ${ }^{25,26}$. A entrevista com os pais leva cerca de 60 minutos. Este estudo utilizou apenas as escalas de habilidades funcionais e de assistência do cuidador que avaliam a área de mobilidade da criança. Para a análise dos dados foram usados os escores totais brutos destas duas escalas. O teste PED|24,29 foi utilizado para avaliar as habilidades e a independência na função de mobilidade das crianças, aos 12 meses de idade (corrigida para crianças do grupo de risco).

\section{Procedimentos}

Crianças de ambos os grupos foram avaliadas por dois examinadores que foram previamente treinados na aplicação dos dois testes e a consistência entre os mesmos foi avaliada obtendo-se boa confiabilidade, indicada por baixos índices de variabilidade entre-examinadores (coeficiente de variação variando de 0,01 a 0,028).

Antes da inclusão das crianças neste estudo, os pais ou responsáveis foram informados sobre os objetivos e procedimentos e assinaram um termo de consentimento para a participação de seu filho. Crianças do grupo de risco foram avaliadas no serviço ACRIAR que funciona no Ambulatório Bias Fortes do Hospital das Clínicas, UFMG. As crianças do grupo controle foram avaliadas em cre-

Tabela 1. Comparação da função motora de crianças dos grupos de risco e controle, aos 8 e 12 meses de idade (teste t Student).

\begin{tabular}{|c|c|c|c|c|c|}
\hline Idade & $\mathrm{t}$ & $\mathrm{p}$ & Média & $\begin{array}{c}\text { Erro } \\
\text { padrão }\end{array}$ & \\
\hline \multicolumn{6}{|l|}{8 meses } \\
\hline \multirow[t]{2}{*}{ Grupos: } & Controle & & & 31,31 & 1,77 \\
\hline & Risco & 0,131 & 0,897 & 30,94 & 2,27 \\
\hline \multicolumn{6}{|c|}{12 meses (Habilidade) } \\
\hline \multirow[t]{2}{*}{ Grupos: } & Controle & & & 26,44 & 1,89 \\
\hline & Risco & 0,229 & 0,821 & 25,88 & 1,58 \\
\hline \multicolumn{6}{|c|}{12 meses (Independência) } \\
\hline \multirow[t]{2}{*}{ Grupos: } & Controle & & & 16,00 & 1,30 \\
\hline & Risco & $-1,235$ & 0,227 & 18,13 & 1,15 \\
\hline
\end{tabular}

ches da região metropolitana de Belo Horizonte. O procedimento para avaliação destas crianças consistiu de contato com o responsável pela creche, apresentação e esclarecimentos sobre o estudo, solicitação de espaço físico para aplicação dos testes de desenvolvimento e facilitação do contato com os pais das crianças. Por ser estudo com características de acompanhamento longitudinal, as crianças dos dois grupos foram avaliadas aos 8 e novamente aos 12 meses (idades corrigidas para crianças nascidas pré-termo).

Este estudo foi aprovado pelo Comitê de Ética em Pesquisa da UFMG.

Análise estatística

A análise descritiva dos dados informou sobre as características das crianças de cada grupo em relação às seguintes varáveis: sexo, nível sócio-econômico da família, peso ao nascimento e idade gestacional. $\mathrm{Na}$ análise inferencial dos dados, foi utilizado o teste t de Student para grupos independentes para avaliar diferença entre as médias dos escores dos dois grupos aos 8 e aos 12 meses de idade. O índice de correlação Pearson ( $r$ ) foi utilizado para avaliar a relação entre desempenho motor aos 8 meses e mobilidade aos 12 meses, em cada grupo. Para as análises inferenciais foi considerado o índice de significância $\alpha=0,05$.

\section{RESULTADOS}

No grupo de risco, a idade gestacional e o peso ao nascimento das crianças variaram de 26 a 34 semanas (média: 30,25 sem.; desvio padrão: 2,54 sem.) e de 770 a 2240 gramas (média: 1293g; desvio padrão: $435 \mathrm{~g}$ ), respectivamente. Este grupo foi composto por 11 crianças do sexo feminino e cinco crianças do sexo masculino. O nível sócio-econômico das famílias variou de classe $B 2 \mathrm{a} D$, predominando a classe C (nível sócio-econômico baixo) ${ }^{27}$.

No grupo controle, todas as crianças apresentaram idade gestacional superior a 37 semanas e peso ao nascimento maior que 2500 gramas. Este grupo incluiu 11 crianças do sexo feminino e cinco crianças do sexo masculino. Em relação ao nível sócio-

Tabela 2. Matriz de correlação da função motora de crianças do grupo controle $(n=16)$.

\begin{tabular}{lccc}
\hline & $\begin{array}{c}\text { Movimentação } \\
\text { espontânea aos } \\
8 \text { meses }\end{array}$ & $\begin{array}{c}\text { Habilidades } \\
\text { de mobilidade } \\
\text { aos } 12 \text { meses }\end{array}$ & $\begin{array}{c}\text { Independência } \\
\text { em mobilidade } \\
\text { aos } 12 \text { meses }\end{array}$ \\
\hline $\begin{array}{l}\text { Movimentação espontânea } \\
\text { aos 8 meses }\end{array}$ & $\begin{array}{l}\mathrm{p}=1,00 \\
\mathrm{H}=-\end{array}$ & & \\
Habilidades de mobilidade & $r=0,68^{*}$ & $r=1,00$ & \\
aos 12 meses & $p=0,004$ & $p=-$ & \\
Independência em mobilidade & $r=0,04$ & $r=0,25$ & $r=1,00$ \\
aos 12 meses & $p=0,877$ & $p=0,350$ & $p=-$ \\
\hline
\end{tabular}

*índice de correlação Pearson r estatisticamente significativo (teste não direcional). 
Tabela 3. Matriz de correlação da função motora de crianças do grupo controle $(n=16)$.

\begin{tabular}{lccc}
\hline & $\begin{array}{c}\text { Movimentação } \\
\text { espontânea aos } \\
8 \text { meses }\end{array}$ & $\begin{array}{c}\text { Habilidades } \\
\text { de mobilidade } \\
\text { aos } 12 \text { meses }\end{array}$ & $\begin{array}{c}\text { Independência } \\
\text { em mobilidade } \\
\text { aos } 12 \text { meses }\end{array}$ \\
\hline $\begin{array}{l}\text { Movimentação espontânea } \\
\text { aos 8 meses }\end{array}$ & $\begin{array}{l}\mathrm{p}=1,00 \\
\mathrm{p}=-\end{array}$ & & \\
Habilidades de mobilidade & $r=0,07$ & $r=1,00$ & \\
aos 12 meses & $p=0,814$ & $p=-$ & \\
Independência em mobilidade & $r=0,23$ & $r=0,80 *$ & $r=1,00$ \\
aos 12 meses & $p=0,397$ & $p=0,001$ & $p=-$ \\
\hline
\end{tabular}

*Índice de correlação Pearson r estatisticamente significativo (teste não direcional).

econômico das famílias, este grupo variou de B2 a $D$, sendo a classe $C$ a mais frequente ${ }^{27}$.

A Tabela 1 informa sobre os valores do test T das comparações entre grupos, aos 8 e 12 meses de idade, com as respectivas médias dos escores obtidos pelo grupo.

Resultados das análises dos testes t não revelaram diferenças significativas no desempenho motor aos 8 meses, nas habilidades de mobilidade aos 12 meses e nem na independência em mobilidade aos 12 meses de idade.

As Tabelas 2 e 3 apresentam as matrizes de correlação dos resultados das análises da relação entre a desempenho motor aos 8 meses e a mobilidade (habilidade e independência) e o desempenho aos 12 meses, nos dois grupos. No grupo controle, estes resultados revelam uma relação significativa $(p=0,004)(r=0,67)$ entre desempenho motor aos 8 meses e habilidades de mobilidade aos 12 meses (Tabela 2). No grupo pré-termo, a única relação significativa $(p=0,0001)(r=0,80)$ foi entre as habilidades de mobilidade e a independência em mobilidade aos 12 meses de idade (Tabela 3).

\section{DISCUSSÃO}

Dois resultados apresentados neste estudo merecem ser destacados. Em primeiro lugar, não foi observada diferença significativa entre os grupos de risco e controle nas variáveis referentes à função motora avaliadas aos 8 e aos 12 meses de idade, lembrando-se que se trata de pré-termos sem outros distúrbios e com idade corrigida. Tal resultado parece contradizer um grande número de estudos que vêm documentando diferenças entre crianças que apresentaram sinais de risco ao nascimento e crianças que não tiveram intercorrências neonatais. Entretanto, análise mais específica desta literatura sugere que os resultados deste estudo podem estar complementando a literatura existente.
Diversos estudos têm comparado o desempenho motor de crianças de risco com o de crianças típicas, priorizando componentes da postura e do movimento, tais como tônus, amplitude de movimentação, reflexos primitivos e manutenção de postura antigravitacional $\left.\right|^{1-3,11,15,16}$. Alguns autores compararam características do desenvolvimento motor em crianças nascidas com baixo peso e crianças do grupo controle ${ }^{2,11}$. Estes autores identificaram diferenças significativas entre os grupos: crianças do grupo de baixo peso apresentaram tônus mais baixo e pobre desempenho motor grosseiro com atraso no desenvolvimento postural e no controle de cabeça. Similarmente, estudos que compararam as mesmas características motoras em crianças nascidas a termo e pré-termo observaram que o nascimento prétermo parece estar associado a tônus muscular mais baixo, pobre controle postural e reflexos primitivos menos evidentes ${ }^{1,11,15,16,18}$. Estes estudos documentaram diferenças em alguns fatores relacionados a componentes neuromotores do movimento de crianças de risco, comparadas com crianças típicas. $\mathrm{O}$ presente estudo analisou características diferentes daquelas já documentadas na literatura. Ao invés de componentes neuromotores, este estudo investigou o impacto do nascimento pré-termo em variáveis relacionadas com movimentação espontânea da criança (8 meses), bem como em habilidades e independência na realização de transferências funcionais e na locomoção (12 meses). Uma hipótese sobre a relação entre os resultados deste estudo e os resultados apresentados na literatura sugere que crianças de risco e crianças sem intercorrências neonatais podem apresentar diferenças significativas na caracterização dos componentes neuromotores do movimento, mas, apesar disso, é possível que estas diferenças não sejam evidentes quando se observa a realização de funções de transferência e de locomoção. Com base nesta hipótese, pode-se 
argumentar que apesar das alterações nos componentes neuromotores observadas em crianças de risco, as mesmas acabam desenvolvendo estratégias motoras que lhes permitem desempenhar atividades funcionais como as crianças do grupo controle. Alguns autores vêm apresentado evidências que podem dar suporte a tal argumentação $13,21,23,31,32$. Einspieler et al. ${ }^{31}$ compararam o desempenho motor de crianças de risco com o de crianças típicas e encontraram diferenças na qualidade da movimentação espontânea, mas não na quantidade de mobilidade das crianças. É importante ressaltar que tal argumentação permanece como uma hipótese que deve ser testada em investigações futuras.

Uma outra hipótese sobre a relação entre os resultados deste estudo e as evidências apresentadas na literatura pode estar associada a diferenças na caracterização dos grupos de risco. Parte da literatura centra suas investigações em crianças denominadas pré-termo extremo, ou seja, aquelas nascidas com muito baixo peso (peso ao nascimento inferior a 1500 gramas) ou baixo peso extremo (peso inferior a 800 gramas) e crianças com idade gestacional

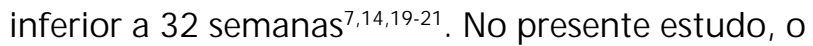
grupo de risco incluiu crianças com caraterísticas de peso e idade gestacional ao nascimento bastante variadas. É possível que diferenças no desenvolvimento de crianças pré-termo e a termo sejam mais evidentes quando se consideram crianças com características de risco perinatal extremas.

A generalização deste primeiro resultado pode estar prejudicada devido às características das crianças que compuseram o grupo de risco deste estudo. Estas crianças participam de um serviço ambulatorial de acompanhamento de crianças de risco (ACRIAR) onde fazem avaliações periódicas do crescimento e desenvolvimento e cujos pais ou responsáveis são orientados por profissionais de uma equipe interdisciplinar. Estas orientações geralmente informam sobre maneiras específicas de estimular o desenvolvimento da criança, com base nos dados coletados nas avaliações. É possível que tais orientações possam ter influenciado o ritmo de desenvolvimento das crianças do grupo de risco e, indiretamente, ter estimulado as funções motoras destas crianças. Futuros estudos devem investigar o impacto de orientações dadas aos pais de crianças de risco no desenvolvimento da função de mobilidade.

Um segundo resultado que merece ser discutido refere-se às associações significativas observadas entre variáveis deste estudo. No grupo controle foi evidenciada relação significativa entre avaliação da movimentação espontânea aos 8 meses e habilidades de mobilidade aos 12 meses. Tal resultado sugere que a movimentação da criança no terceiro trimestre de vida parece interferir na capacidade da mesma de realizar transferências funcionais e de locomover-se no ambiente aos 12 meses de idade. A relação observada entre estas variáveis indica que, além da movimentação espontânea, outros fatores podem interferir na mobilidade destas crianças ao final do primeiro ano. Alguns estudos vêm demonstrando a existência de associação entre movimentação voluntária e função motora como fatores preditivos na detecção do atraso no desenvolvimento motor. Darrah et al. ${ }^{29}$ investigaram a validade do teste AIMS utilizando outros testes padronizados tradicionais de desenvolvimento infantil e, segundo estes autores, o AIMS demonstrou melhor especificidade preditiva aos 8 meses que os outros testes.

No grupo de risco, os resultados mostraram associação significativa entre a capacidade da criança de realizar transferências funcionais e de locomoverse no ambiente e o uso de forma independente destas capacidades na sua rotina diária. Tal resultado sugere que, ao desenvolver estas habilidades motoras, crianças do grupo de risco tendem a incorporar tais habilidades em seu repertório funcional. Estes resultados sugerem ainda que os cuidadores das crianças deste grupo permitem que as mesmas façam uso das habilidades desenvolvidas, realizando tarefas de transferência e mobilidade com independência. Por outro lado, a associação entre movimentação espontânea aos 8 meses e mobilidade aos 12 meses, neste grupo de crianças, não foi significativa como observado no grupo controle. Este resultado pode ser atribuído ao fato dos dois testes utilizados neste estudo avaliarem a função motora da criança com ênfase diferenciada. O teste AIMS utilizado aos 8 meses avaliou a qualidade da movimentação da criança, enfatizando características como descarga de peso, postura e movimentos antigravitacionais. Aos 12 meses, o teste PEDI avaliou a capacidade da criança para realizar determinada tarefa motora como andar, subir escada e fazer transferências funcionais. O teste PEDI informa sobre o desempenho de atividades de mobilidade frequentemente executadas no cotidiano das crianças e ainda sobre a quantidade de ajuda fornecida pelo cuidador. A qualidade com que a criança desempenha as atividades de mobilidade não é diretamente documentada por este teste. Portanto, é possível que estudos que utilizem instrumentos que priorizam a qualidade da movimentação da criança no desem- 
penho de atividades de mobilidade típicas de sua rotina diária possam obter resultados diferentes dos apresentados por este estudo.

Concluindo, este estudo apresenta informações importantes sobre o desenvolvimento da função motora em crianças de risco e crianças sem intercorrências perinatais. $O$ desenho longitudinal reforça os resultados apresentados, uma vez que investigações longitudinais são mais adequadas para subsidiar inferências sobre mudanças no desenvolvimento infantil.

Agradecimentos - Nossos agradecimentos à equipe do ACRIAR do complexo do Hospital das Clínicas da UFMG e às creches da região metropolitana de Belo Horizonte por facilitarem o contato com os pais, viabilizando o desenvolvimento do estudo.

\section{REFERÊNCIAS}

1. Howard J, ParmeleeAH, Kopp CB, Littman B. A neurologic comparison of pre-term and full-term infants at term conceptional age. J Pediatr 1976;88:995-1002

2. Jeng SF, Yau KT, Teng RJ. Neurobehavioral development at term in very low-birthweight infants and normal term infants in Taiwan. Early Hum Dev 1998;51:235-245.

3. Mancini MC, Paixão ML, Gontijo AB, Ferreira AA. Perfil do desenvolvimento neuromotor do bebê de alto risco no primeiro ano de vida. Temas sobre Desenvolvimento 1992;8:3-8.

4. Marcondes E. Pediatria básica 7.Ed. São Paulo: Savier, 1995.

5. Jeng S, Yau KT, Chen L, H siao S. A Iberta infant motor scale: reliability and validity when used on preterm infants in Taiwan. Phys Ther 2000; 80:168-178.

6. Jornal do Comércio on line: $5^{\mathrm{a}}$ Jornada Materno-infantil. Jornal do Comércio; Recife, 1998. [Disponível em: http:/ / www.2.uol.com.br/ JC/ 1998/ 1810/ fa1810c.html. A cesso em: 15 de maio, 2001]

7. Klaus MH, Fanaroff AA. Alto risco em neonatologia 2.Ed. Rio de Janeiro: Interamericana, 1982.

8. Molteno $C$, Grosz $P$, Wallace $P$, Jones $M$. Neurological examination of the preterm and full-term infant at risk for developmental disabilities using the Dubowitz neurological assessment. Early Hum Dev 1995; 41:167-176

9. Magalhães LC, Barbosa VM, Paixão ML, Figueiredo EM, Gontijo APB. A companhamento ambulatorial de recém-nascidos de alto-risco: características da população atendida e incidência de sequelas funcionais. Rev Paul de Pediatr 1998;16:191-196.

10. Paixão ML, Mancini MC, Figueiredo EM, Ferreira APA, Gontijo APB O impacto da relação peso-idade gestacional no desenvolvimento do bebê prétermo. Temas sobre Desenvolvimento 1994;3:54-60.

11. Mancini MC, Vieira FL. Desenvolvimento motor em crianças nascidas com baixo peso: uma revisão da literatura. Temas sobre Desenvolvimento 2000;9:21-24.
12. Piper MC, Byrne PJ, Darrah J, Watt MJ. Gross and fine motor development of preterm infants at eight and 12 months of age. Dev Med Child Neurol 1989;31:591-597.

13. Allen MC, Alexander GR. Using motor milestones as a multistep process to screen preterm infants for cerebral palsy. Dev Med Child Neurol 1997; 39:12-16.

14. Allen MC, Capute AJ. Neonatal neurodevelopmental examination as a pre dictor of neuromotor outcomein prematureinfants. Pediatrics 1989; 83:498-506.

15. Forslud M, Bjerrel. N eurological assessment of preterm infants at term conceptional age in comparison with normal full-term infants. Early Hum Dev 1983;8:195-208.

16. GrootL, HopkinsB, Touwen B. Motor assymmetries in preterm infantsat 18 weeks of correct ageand outcomes at 1 year. Early Hum Dev 1997;48:35-46.

17. Heriza CB. Comparison of leg movements in preterm infants at term with healthy full-term infants. Phys Ther 1988;68:1687-1693.

18. Persson K, Stromberg B. Structured observation of motor performance applied to preterm and full-term infants who needed neonatal intensive care: a cross-sectional analysis of progress and qualitity of motor performance at ages 0-10 months. Early Hum Dev 1995;43:205-224.

19. Piper MC, Kunos I, Willis DM, Mazer B. Effect of gestational age on neurological functioning of the very low-birthweight infant at 40 weeks. Dev Med Child Neurol 1985; 27:596-605.

20. Piper MC, Kunos VI, Willis DM, Mazer BL, Ramsay M, Silver KM. Early physical therapy effects on the high-risk infant: a randomized controlled trial. Pediatrics 1986;78:216-224.

21. Piek JP, Carman R. Developmental profiles of spontaneous movements in infants. Early Hum Dev 1994;39:109-126.

22. Piper MC, Darrah J. Motor assesment of the developing infant. Philadelphia: WB Saunders, 1994.

23. Prechtl HFR, Cioni G, Ferrari F, Paolicelli B, Einspieler C, Roversi MF. Which better predictslater outcomein fullterminfants: quality of general movements or neurological examination? Early Hum Dev 1997;50:71-85.

24. Mancini, MC. A valiando o desenvolvimento neuromotor: uma análise crítica. Arq de N europsiquiatria 2001;59(Supl.1):33-34.

25. Haley SM, Coster WJ, Ludlow LH, Haltiwanger JT, Andrellow PJ. Pediatric evaluation of disability inventory: development, standardization and administration manual. Boston: New England Medical Center; 1992:1-300.

26. Nichols DS, Case-Smith J. Reliability and validity of the pediatric evaluation of disability inventory. Pediatr Phys Ther 1996;8:15-24.

27. Associação Brasileira dos Institutos de Pesquisa de Mercado (ABIPEME). Critério declassificação econômica brasil. [Disponível em: [http:/ / www.targetmark.com.br/ bf2001/ br/ criterio.htm. A cesso em: 15 de julho, 2001.]

28. Long TM, Tieman B. Review of two recently published measurement tools: the AIMS and the T.I.M.E. Pediatr Phys Ther 1998;10:62-66.

29. Darrah J, Piper MC, Watt M. Assessment of gross motor skills of at risk infants: predictive validity of the alberta infant motor scale. Dev Med Child Neurol 1998; 40:485-491.

30. Haley SM, Coster WJ, Ludlow LH, Haltiwanger JT, Andrellow PJ. Inventário de avaliação pediátrica de disfunção: versão brasileira. Mancini MC. Belo Horizonte: Laboratórios de Atividade e Desenvolvimento Infantil UFMG, 2000.

31. Einspieler C, Prechtl HFR, Ferrari F, Cionib G, Bos AF. The qualitative assessment of general movements in preterm, term and young infants: review of the methodology. Early Hum Dev 1997;50:47-60.

32. Heriza CB. Organization of leg movements in preterm infants. Phys Ther 1988;68:1340-1346. 